\title{
Kernos
}

Revue internationale et pluridisciplinaire de religion grecque antique

$10 \mid 1997$

Varia

\section{TÖRÖK, Hellenistic and Roman Terracottas from Egypt}

Michel Malaise

URL : http://journals.openedition.org/kernos/696

DOI : $10.4000 /$ kernos.696

ISSN : 2034-7871

Éditeur

Centre international d'étude de la religion grecque antique

Édition imprimée

Date de publication : 1 janvier 1997

Pagination : 368-371

ISSN : 0776-3824

Référence électronique

Michel Malaise, "L. TöRöк, Hellenistic and Roman Terracottas from Egypt », Kernos [En ligne], 10 | 1997, mis en ligne le 12 avril 2011, consulté le 23 septembre 2020. URL : http://journals.openedition.org/ kernos/696 ; DOI : https://doi.org/10.4000/kernos.696 
Laslo TöRöк, Hellenistic and Roman Terracottas from Egypt, Roma, «L'Erma » di Bretschneider, 1995. 1 vol. in $-4^{\circ}$, 199 p., 173 pl., dont 16 en couleurs (Bibliotbeca archaeologica, 15. Monumenta antiquitatis extra fines Hungaricae reperta, vol. IV). Prix : US\$ 315.

Ce luxueux livre constitue une magnifique étude des terres cuites gréco-romaines d'Égypte conservées au Musée des Beaux-Arts de Budapest. L'A. contribue ainsi à enrichir un corpus et à alimenter la réflexion sur une production qui, au cours des dernières années, a fortement retenu l'attention des chercheurs, comme nous l'avons rappelé dans le compte rendu que nous consacrons dans ce même numéro au livre de Jutta FISCHER. L'ouvrage de L. TöröK se veut d'abord un catalogue qui décrit chaque pièce, reproduite en fin de volumes grâce à des planches de grande qualité, avec une extrême minutie, présentant chacune de ces figurines comme une œuvre d'art individuelle, avec ses traits spécifiques. Chaque notice s'attache longuement à des considérations stylistiques et iconographiques afin de tenter de déterminer l'époque de la création du type en cause et de dater la fabrication de l'objet étudié. Si l'A. ne présente pas une discussion d'ensemble sur les conditions du contexte historico-religieux, les problèmes relatifs à la signification et à l'utilisation de chaque figurine sont soigneusement abordés. La perspective adoptée diffère donc quelque peu de celle retenue par J. FISCHER qui offre d'abord une vision diachronique de la typologie des terres cuites. Après avoir défini l'esprit du catalogue de Budapest, nous pouvons à présent voir de plus près ce qu'il contient.

Après une préface (p. 9-12) de J.G. SZILAGYI retraçant l'histoire de la collection, l'A. nous livre une introduction (p. 17-25) articulée en quatre temps. Il nous présente d'abord le matériel constitué de 326 pièces, dont la presque totalité est conservée au Musée de Budapest. Ces figurines proviennent de l'ancienne collection P. Arndt, de collections privées hongroises et d'achats effectués au Caire. Il en résulte que l'on ignore, comme le plus souvent d'ailleurs, la provenance précise de ces terres cuites que dès lors, seule, une fine analyse stylistique peut nous aider à dater. On soulignera que les figurines ici étudiées sont totalement inédites ou n'avaient donné lieu qu'à une mention laconique et dépourvue d'illustration.

L'A. aborde ensuite les difficiles questions relatives à la fabrication, la provenance et les ateliers. Peu de données, à l'exception de traits stylistiques particuliers, permettent de reconnâtre les ateliers. Si les couleurs se révèlent sans intérêt, on distinguera deux sortes de terre : une belle argile importée et considérée généralement comme utilisée dans les officines d'Alexandrie et un limon du Nil, allant du rouge au brun, caractéristique des productions que l'on qualifie du terme vague de "fayoumique ». La détermination des ateliers est d'autant plus malaisée que l'examen des trouvailles exécutées in situ à Karanis et Tell Atrib montre qu'un même centre de fabrication a réalisé des œuvres qui couvrent tout l'éventail de la production de l'Égypte hellénistique et romaine. Il convient en outre de ne pas oublier que moules et figurines ont pu voyager.

La troisième partie de l'introduction traite les questions d'iconographie et commence par nous mettre en garde contre le danger que constituent, pour retracer l'évolution de la religiosité dans l'Égypte ptolémaïque et romaine, des datations peu assurées. Pour L.T., trop de figurines ont été datées de l'époque romaine, alors que bien des représentations divines remontent à l'époque hellénistique et doivent être mises en 
relation avec Alexandrie. On soulignera que ce «vieillissement » rejoint les propositions de J. FISCHER. Suivent des intéressantes observations sur la signification qu'il convient d'attribuer aux « grotesques ». En accord avec les travaux de N. HimmelmanN et de H.P. LAubSCHER, l'A. refuse de voir dans ces figurines une illustration de difformités physiques réelles, mais les considère plutôt comme un produit du réalisme social hellénistique et du mépris affiché à l'égard des classes inférieures et de leurs coutumes religieuses. Le seul réalisme alexandrin ne suffit cependant à rendre compte de tous ces « grotesques » et la dimension religieuse est parfois loin d'être négligeable. Tel est le cas des desservants dionysiaques dont l'allure caricaturale ne peut évidemment être mise sur le compte de l'hostilité des Grecs. L'émergence des grotesques phalliques doit aussi être replacée dans le contexte memphite du culte de Ptah-Sokaris-Osiris, associé à Harpocrate et Bès, et procède donc de racines pré-ptolémaïques. L'imagerie d'Harpocrate est encore l'objet d'observations importantes. Il est proposé de faire remonter des types habituellement considérés comme romains à la haute et moyenne époque hellénistique $\left(c f . \mathrm{n}^{\circ} 56-59,75,77,81,91\right)$. L'A. considère même que tous les principaux types de l'iconographie d'Harpocrate dérivent de prototypes créés dès le III $^{\mathrm{e}}$ siècle et que le rôle joué par Alexandrie en ce domaine fut essentiel, tant sur le plan cultuel que des points de vue iconographique et stylistique (p. 71). L'extraordinaire popularité d'Harpocrate ne peut tenir à son seul rôle de protecteur des enfants. Pour expliquer cette vogue, selon L.T., sans oublier ses prérogatives claires de dieu de la fertilité, il ne faut pas négliger les connotations funéraires du dieu-enfant phallique qui assure la survie à cause de ses vertus procréatrices, ni ses liens avec l'idéologie de la légitimité royale des Ptolémées, dans la mesure où comme héritier de son père, il constitue un modèle pour le nouveau souverain. On notera encore une remarque, à première vue curieuse, à propos de l'iconographie de Déméter qui offre deux types: l'un ( $\mathrm{n}^{\circ} 31$ ), contaminé par Isis, serait le reflet de la politique des premiers Ptolémées, tandis que le second ( $\left.n^{\circ} 29\right)$, qui n'offre aucun lien avec la déesse égyptienne, serait de la fin de l'époque hellénistique. Bien des types montrent la complexité des modèles créés à la fin du $\mathrm{III}^{\mathrm{e}} \mathrm{s}$. ou au $\mathrm{II}^{\mathrm{e}}$ siècle Un fort bel exemple nous est fourni par un vase plastique $\left(n^{\circ} 145\right)$ qui représente une prêtresse nubienne vêtue d'un costume égyptien et accomplissant un rite grec, probablement une libation chthonienne.

La dernière partie de l'introduction s'attache aux problèmes de datation et de style. Si les caractéristiques stylistiques sont, aujourd'hui encore, essentielles pour tenter de dater une figurine, puisque l'on ignore la provenance précise de la majorité des objets, pareille entreprise est d'autant plus délicate qu'un même moule pouvait servir longtemps, être retouché, voire reproduit au départ d'une figurine. Cette longévité semble indiquer que l'apparition de nouveaux types n'entraînait pas la disparition d'anciens modèles, phénomène traduisant bien la permanence iconographique des concepts religieux. En présence d'une figurine, il convient donc d'essayer de déterminer l'époque de la création du prototype et la date de la production de cette figurine. Pour ce faire, on recourra à une comparaison avec des terres cuites émanant d'autres provinces hellénistiques, sans minimiser le rôle innovateur d'Alexandrie. On ne perdra pas non plus de vue que les ateliers provinciaux ont dû rester plus conservateurs, tandis que les grands centres comme Alexandrie étaient naturellement plus ouverts aux influences extérieures. L'A. souligne enfin que le monde des terres cuites égyptiennes reflète une interpénétration des composantes égyptiennes et grecques, une remarque essentielle sur les effets de la coexistence sur les rives du Nil entre Égyptiens et Grecs, 
que l'on a trop souvent imaginés vivant dans deux mondes hermétiquement clos. L'étude de l'hellénisation d'Isis à laquelle nous venons de nous livrer, pour l'introduction du catalogue consacré à une prochaine exposition qui se tiendra à Milan et réservée à Isis, nous a convaincu de la même réalité. Certains sujets égyptiens apparaissent même comme des adaptations de modèles étrangers ( $c f$. p. 53, 62, 78, 108-109).

Le catalogue est structuré, comme d'habitude, selon une présentation fondée sur les types iconographiques. La première section est consacrée au panthéon ( $\left.n^{\circ} 1-132\right)$. Le lecteur y trouvera les divinités suivantes: Aphrodite $\left(n^{\circ} 4-5\right)$, Apis $\left(n^{\circ} 6\right)$, Athéna $\left(n^{\circ} 7-9\right)$, Bès et membres de sa famille ( $\left.n^{\circ} 10-28\right)$, Déméter $\left(n^{\circ} 29-31\right)$, Dionysos $\left(n^{\circ} 32\right)$, Dioscures $\left(n^{\circ} 33-34\right)$, Éros $\left(n^{\circ} 35-47\right)$, figures phalliques $\left(n^{\circ} 48-53\right)$, Harpocrate $\left(n^{\circ} 54-\right.$ 99); Hathor ( $\left.{ }^{\circ} 100-101\right)$, Hermès-Thot ( $\left.n^{\circ} 102-103\right)$, Isis ( $\left.n^{\circ} 104-108\right)$, Osiris-Canope $\left(n^{\circ} 110-114\right)$, Priape ( $\left.{ }^{\circ} 115-118\right)$, Sérapis ( $\left.n^{\circ} 119-121\right)$, Agathos Daimon ( $\left.n^{\circ} 122-123\right)$, sphinx $\left(\mathrm{n}^{\circ} 124-129\right)$, Thot $\left(\mathrm{n}^{\circ} 130-131\right)$, Zeus-Sarapis $\left(\mathrm{n}^{\circ} 132\right)$. De cette simple énumération, il ressort que, comme dans les autres collections résultant largement du hasard, Harpocrate se taille la part du lion.

La seconde section regroupe les types empruntés à la mythologie classique : Silène $\left(n^{\circ} 133-138\right)$, Pan et Nymphe ( $\left.n^{\circ} 139\right)$, fragment d'un bouclier votif avec gorgoneion $\left(n^{\circ} 140\right)$, Sirène $\left(n^{\circ} 141\right)$. On notera la popularité des thèmes dionysiaques qui s'explique dans la mesure où les Lagides se sont identifiés à ce dieu.

La section III rassemble des figurines dans l'exercice d'activités cultuelles : prêtres portant Harpocrate ( $n^{\circ} 142-143$ ), prêtres, desservants et musiciens ( $n^{\circ} 144-168$ ), dont une intéressante scène de cuisine ( $\left.\mathrm{n}^{\circ} 159\right)$, qui, à notre sens, éclaire parfaitement la signification des Harpocrates touillant dans un pot, comme nous pensons l'avoir établi ailleurs ( $c f$. M. MALAISE, Harpocrate au pot, in Religion und Pbilosopbie im alten Ägypten. Festgabe für Pb. Derchain, Louvain, 1991, p. 219-232; ID., Questions d'iconographie barpocratique soulevées par des terres cuites d'Égypte gréco-romaine, in Hommages à J. Leclant, III, Le Caire, 1994, p. 373-383).

Dans une quatrième section, sont traitées les terres cuites illustrant la piété personnelle: orants et orantes ( $\left.n^{\circ} 169-181\right)$; pseudo-Baubo ( $\left.{ }^{\circ} 182-190\right)$, que L.T., malgré d'autres hypothèses à nos yeux plus séduisantes, continue à mettre en relation avec les mystères éleusiniens au cours desquels aurait été exprimée l'identification de Déméter avec Isis et de Dionysos avec Osiris; têtes féminines ( $\left.{ }^{\circ} 191-193,201\right)$; garçons $\left(n^{\circ}\right.$ 194-200); figurine funéraire $\left(n^{\circ} 202\right)$; ex-voto de fertilité (?) de femmes nues debout (203-209). Viennent ensuite des femmes nues, jambes jointes et bras collés contre le corps ( $\left.\mathrm{n}^{\circ} 210-211\right)$ dans lesquels l'A. refuse de voir des images divines à cause de leur nudité, même si ces femmes sont parfois coiffées d'un polos coiffé de la couronne isiaque du basileion; il préfère y reconnaître des figurines de fertilité déposées comme ex-voto dans le temple d'Hathor. Enfin, une scène de symplegma ( $\left.\mathrm{n}^{\circ} 212\right)$ est interprétée, à cause de la couronne florale des partenaires, comme une offrande votive que des couples pouvaient déposer dans un temple pour obtenir la fertilité.

Les scènes de genre, représentations d'acteurs, et « grotesques » font l'objet de la section V ( $\left.n^{\circ} 213-263\right)$. Il y est suggéré que les représentations de danseurs et de musiciens grotesques sont à mettre en liaison avec les participants du thiase dionysiaque, tout en constituant une adaptation des traditions égyptiennes concernant le rôle rituel des nains. 
Les animaux sont analysés dans la section VI ( $\mathrm{n}^{\circ}$ 264-286). Sans exclure la possibilité, pour de rares exemplaires, qu'il puisse s'agir de jouets $\left(n^{\circ} 273\right)$, L.T. préfère situer le rôle de ces petits animaux dans un climat religieux : substituts pour sacrifices d'animaux dans les temples (porc, coq) ou ex-voto. Le thème du chien est mis en rapport avec la chienne personnifiant l'étoile Sothis et qu'Isis, productrice de la crue, peut enfourcher à partir de l'époque romaine; l'animal devient ainsi signe de fertilité. Sans dénier la pertinence de cette exégèse, nous avons montré ailleurs que le chien est susceptible de multiples connotations ( $c f$. M. MALAISE, Harpocrate, la tortue et le cbien. Contribution à l'iconographie du fils d'Isis, in Bulletin de la Société Francaise d'Égyptologie, 122 [1991], p. 13-35). L'image du tilapia est, elle, assurément symbole d'auto-génération, et donc de renaissance, selon une ancienne conception égyptienne.

Enfin, les sections VII et VIII sont, respectivement, consacrées à quelques lanternes en forme de naiscos $\left(\mathrm{n}^{\circ} 287-291\right)$ et à des varia $\left(\mathrm{n}^{\circ} 292-321\right)$. Parmi ces derniers sont particulièrement dignes d'attention un naiscos monté sur quatre roues, et dont la porte est flanquée d'amphores, $\left(n^{\circ} 292\right)$ imitant les véhicules à roue qui remplacent parfois les barques processionnelles; une kliné avec paysage nilotique $\left(\mathrm{n}^{\circ} 295\right)$ et deux porte-torches miniatures ( $n^{\circ} 305-306$ ) décorés de figures ou de symboles divins.

Pour mettre un terme à notre recension de cet ouvrage fondamental, nous voudrions souligner que l'A. a choisi d'interpréter la quasi totalité des terres cuites étudiées à la lumière de préoccupations religieuses, même pour des objets d'apparence banale et tirés du monde profane. Cette constatation essentielle rejoint les récentes et intéressantes considérations émises par G. NACHTERGAEL, CdE, 79 (1995), p. 263-268. Sur cette question, comme sur bien d'autres, la publication d'un maximum de terres cuites permettra de cerner de mieux en mieux chronologie et signification d'une documentation qui nous plonge de façon extraordinaire et vivante dans le monde vécu et imaginaire de l'Égypte gréco-romaine. L'ouvrage de L.T. est une contribution d'importance à ce travail de restauration.

Michel MALAISE

(Université de Liège)

\section{Reinhold MERKELBACH, Isis regina - Zeus Sarapis. Die griechisch- ägyptische Religion nach den Quellen dargestellt, Stuttgart-Leipzig, 1995.} 1 vol. $19 \times 25,3 \mathrm{~cm}$, XXVIII+722 p., 35 dessins, 252 fig. et 8 pl. couleurs hors-texte. ISBN 3-519-07427-3.

Le titre de cet ouvrage est relativement trompeur car il annonce une synthèse de la religion isiaque, alors qu'en réalité le livre, composé de deux grandes parties bien distinctes, a été écrit pour défendre une thèse que l'A. avait déjà développée dans Roman und Mysterium en 1962. Selon cette thèse, plusieurs romans d'époque impériale seraient en fait des romans à clef, écrits pour des isiaques. Pour donner une plus large base à sa " démonstration », R.M. a entrepris d'écrire ce livre dont la première partie, la plus volumineuse (p. 57-334), nous retrace une vue d'ensemble des cultes isiaques, depuis la haute époque hellénistique jusqu'à leur disparition devant le christianisme triomphant. Le panorama est fondé sur une documentation variée, mais l'A. privilégie documents littéraires et arétalogiques, c'est-à-dire une vision assez érudite et moins proche des réalités quotidiennes que celle obtenue à la lecture des 